\title{
Surface temperature maps for II Peg during 1999-2002
}

\author{
Lindborg, M. ${ }^{1}$, Korpi, M.J. ${ }^{1,4}$, Tuominen, I. $^{1}$, Hackman, T. ${ }^{1}$, Ilyin, $\mathbf{I}^{2}$, \\ Piskunov, N. ${ }^{3}$ \\ ${ }^{1}$ Observatory, PO BOX 14, FI-00014 University of Helsinki, Finland \\ ${ }^{2}$ Astrophysikalisches Institut Potsdam, An der Sternwarte 16, 14482 Potsdam, Germany \\ ${ }^{3}$ Department of Astronomy and Space Physics, Uppsala University, SE-751 20, Uppsala, \\ Sweden \\ ${ }^{4}$ NORDITA, Roslagstullsbacken 23, SE-10691 Stockholm, Sweden
}

\begin{abstract}
The active RS CVn star II Peg has been spectroscopically monitored for almost 18 years with the SOFIN spectrograph at NOT, La Palma, Spain. In this paper we present five new surface temperature maps of the object for the years 1999 (two maps), 2001 (one map) and 2002 (two maps).
\end{abstract}

Keywords. techniques: spectroscopic, stars: late-type, stars: spots

\section{Introduction}

II Peg is one of the very active RS CVn stars and it is the brightest X-ray star with $50 \mathrm{pc}$ of the Sun. RS CVn stars are closely detached binaries where the more massive component is a G-K giant or subgiant and the secondary a subgiant or dwarf of spectral class G to M. Because of the low luminosity of the secondary many RS CVn systems appear as single-line binaries which are suitable for spectral analysis (Berdyugina et al. 1998a). In close binaries the rapid rotation is maintained by tidal forces due to the close companion. Large amplitude brightness variation of RS CVn stars imply the presence of enormous star spots on their surfaces covering up to $50 \%$ of the visible disk. Also coronal $\mathrm{X}$-ray and microwave emissions, strong flares in the optical, UV, radio and X-ray are seen. Cool spots on the stellar surface will locally alter the photospheric absorption lines and continuum intensities.

Previous investigations on the temperature distribution over the surface of II Peg include the study of Berdyugina et al. (1998b), who presented surface temperature maps for 1992-1996, and Bergyugina et al. (1999c) with surface maps for 1996-1999, both studies were based on observations with the SOFIN-spectrograph at NOT. Gu et al. (2003) presented surface images of II Peg for 1999-2001 based on observations with the Coude echelle spectrograph at the Xinglong station of the National Astronomical Observatories, China, but the spectral lines used for inversions were different to that of SOFIN observations. Photometric light curve variations of the object were analysed by Berdyugina \& Tuominen (1998c), and by Rodonò et al. (2000). The results of Berdyugina et al. (1998a,b, 1999a,b,c) and Berdyugina \& Tuominen (1998c) consistently show that there are two active longitudes separated approximately by $180^{\circ}$, migrating in the orbital reference frame, and that a switch of activity level occurs periodically with a period of 4.65 years. In the surface images of $\mathrm{Gu}$ et al. (2003) the general spot pattern was quite similar, but the drift with respect of the orbital frame was less obvious, and the switch of the activity level appeared to occur earlier than predicted by Berdyugina et al (1999c). Rodonò et al. (2000) found a much more complicated spot pattern from 
Table 1. Summary of the observations.

\begin{tabular}{|c|c|c|c|c|c|c|c|}
\hline $\begin{array}{l}\text { HJD } \\
2450000+\end{array}$ & Phase & $\mathrm{S} / \mathrm{N}$ & Label & $\begin{array}{l}\text { HJD } \\
245000+\end{array}$ & Phase & $\mathrm{S} / \mathrm{N}$ & Label \\
\hline $\begin{array}{l}\text { July-August } 1999 \\
1383.7017 \\
1384.7178 \\
1385.7232 \\
1386.7124 \\
1387.7094 \\
1388.7278 \\
1389.7031 \\
1390.7198 \\
1391.7045 \\
1392.7108 \\
1393.7275 \\
1394.7386\end{array}$ & $\begin{array}{l}0.7998 \\
0.9509 \\
0.1004 \\
0.2475 \\
0.3958 \\
0.5472 \\
0.6923 \\
0.8435 \\
0.9899 \\
0.1396 \\
0.2908 \\
0.4411\end{array}$ & $\begin{array}{l}248 \\
238 \\
217 \\
229 \\
200 \\
181 \\
178 \\
151 \\
243 \\
258 \\
189 \\
206\end{array}$ & $\begin{array}{c}9 \\
11 \\
1 \\
3 \\
5 \\
7 \\
8 \\
10 \\
12 \\
2 \\
4 \\
6\end{array}$ & $\begin{array}{l}\text { September } 1999 \\
1443.4928 \\
1443.6184 \\
1444.4788 \\
1445.5656 \\
1447.6084 \\
1448.6212 \\
1449.5979\end{array}$ & $\begin{array}{l}0.6915 \\
0.7102 \\
0.8382 \\
0.9998 \\
0.3036 \\
0.4542 \\
0.5994\end{array}$ & $\begin{array}{l}179 \\
164 \\
230 \\
194 \\
174 \\
184 \\
178\end{array}$ & $\begin{array}{l}4 \\
5 \\
6 \\
7 \\
1 \\
2 \\
3\end{array}$ \\
\hline $\begin{array}{l}\text { August } 2001 \\
2120.5503 \\
2121.5642 \\
2122.7102 \\
2123.6083 \\
2124.5644 \\
2125.6758 \\
2126.7146 \\
2128.6067 \\
2129.6470 \\
2130.6415\end{array}$ & $\begin{array}{l}0.3796 \\
0.5303 \\
0.7008 \\
0.8344 \\
0.9765 \\
0.1418 \\
0.2963 \\
0.5777 \\
0.7324 \\
0.8804\end{array}$ & $\begin{array}{l}192 \\
225 \\
185 \\
196 \\
193 \\
177 \\
189 \\
166 \\
190 \\
208\end{array}$ & $\begin{array}{c}3 \\
4 \\
6 \\
8 \\
10 \\
1 \\
2 \\
5 \\
7 \\
9\end{array}$ & $\begin{array}{l}\text { August } 2002 \\
2507.6078 \\
2508.6582 \\
2509.5877 \\
2510.5909 \\
2511.5413 \\
2512.6316 \\
2513.5494 \\
2514.5854 \\
2515.5768 \\
2518.5749\end{array}$ & $\begin{array}{l}0.9405 \\
0.0967 \\
0.2349 \\
0.3842 \\
0.5255 \\
0.6876 \\
0.8242 \\
0.9782 \\
0.1257 \\
0.5715\end{array}$ & $\begin{array}{l}139 \\
208 \\
200 \\
216 \\
195 \\
212 \\
179 \\
206 \\
207 \\
129\end{array}$ & $\begin{array}{c}9 \\
1 \\
3 \\
4 \\
5 \\
7 \\
8 \\
10 \\
2 \\
6\end{array}$ \\
\hline $\begin{array}{l}\text { November } 2002 \\
2588.3964 \\
2589.3764 \\
2591.3567 \\
2592.3705 \\
2597.4333 \\
2599.4798 \\
2600.4896\end{array}$ & $\begin{array}{l}0.9549 \\
0.1007 \\
0.3951 \\
0.5459 \\
0.2988 \\
0.6031 \\
0.7532\end{array}$ & $\begin{array}{l}207 \\
160 \\
241 \\
219 \\
252 \\
275 \\
259\end{array}$ & $\begin{array}{l}7 \\
1 \\
3 \\
4 \\
2 \\
5 \\
6\end{array}$ & & & & \\
\hline
\end{tabular}

their analysis of photometry: they report on the existence of a longitudinally uniformly distributed component together with three active longitudes, with complicated cyclic behavior. Carroll et al. (2009) have also applied a Zeeman Doppler imaging technique to derive the magnetic field configuration on the surface of II Peg during 2007 using spectropolarimetric (Stokes I and V) observations with SOFIN. Their maps show a very similar spot pattern as found by Berdyugina et al. (1998b, 1999c); moreover, the radial field direction is opposite on different active longitudes.

\section{Observations}

When a star rotates rapidly star spots modify the observed spectral line profiles. As the star rotates, these bumps will move across the absorption line profiles (Hackman et al. (2001); Kurster, M. (1993)). The surface imaging techique is basically to trace these distortions and create a surface map of the star. We use the surface imaging technique developed by Piskunov (the code INVERS7, Piskunov et al. (1990); Piskunov (1991)).

We use the stellar model atmospheres of Kurucz (1993) for local line profile calculations for a set of temperatures and limb angles. This table is then used for the disk 

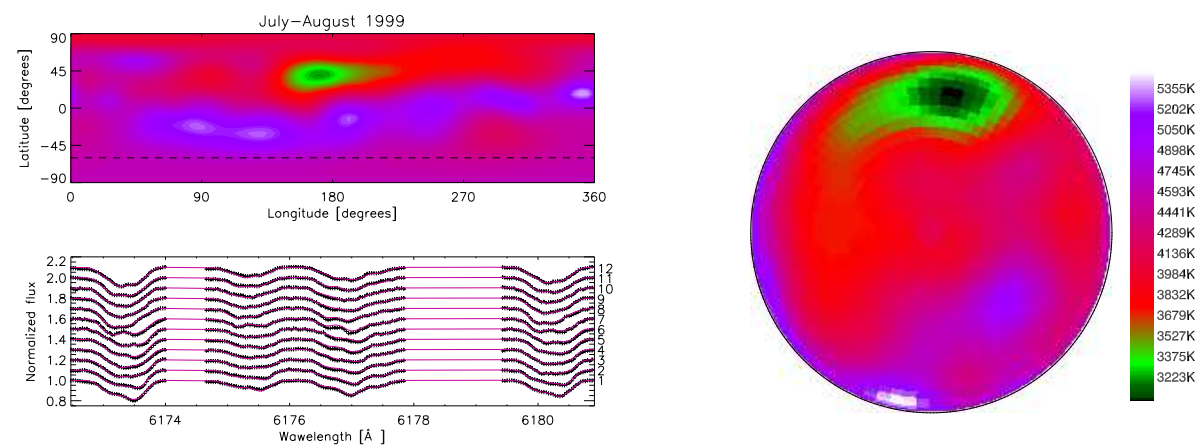

Figure 1. Observing run during July - August 1999. Upper left panel: Mercator projection of the obtained surface temperature distribution. Lower left panel: observed (crosses) and calculated line profiles (solid lines). Panel on the right: Pole-on projection of the surface temperature distribution.
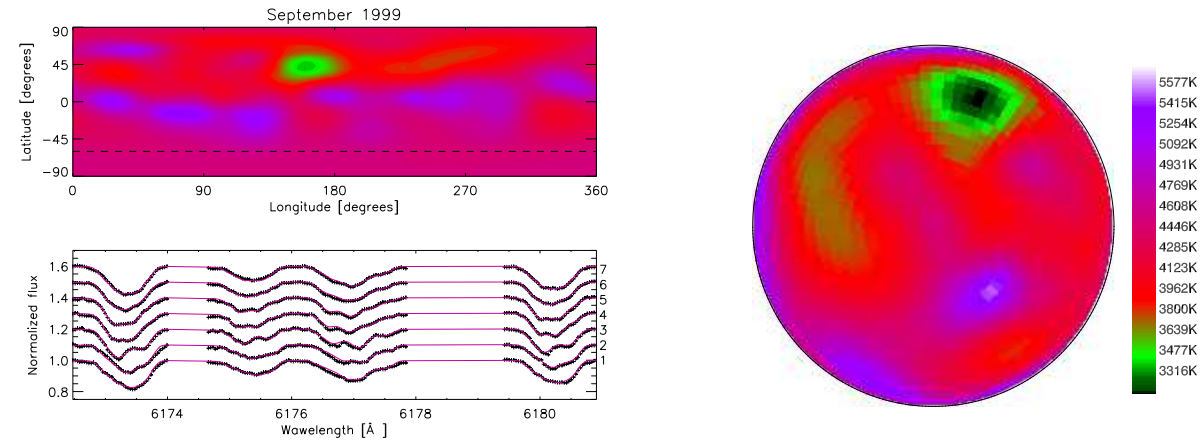

Figure 2. Observing run during September 1999.

integration of a given surface temperature distribution. The surface imaging problem can be solved by searching for a such surface temperature distribution that minimizes the discrepancy function between the observations and the calculated line profiles (Hackman et al. (2001)).

High resolution spectra of II Peg were measured in July-August 1999, September 1999, August 2001, August 2002 and November 2002. All the observations were made using the SOFIN high resolution echelle spectrograph at the $2.6 \mathrm{~m}$ Nordic Optical Telescope (NOT), La Palma, Spain. The data were acquired with the 2nd camera equipped with a CCD detector of $1152 \times 298$ pixels, and the spectral region $6160-6210 \AA$ was chosen for surface imaging. The observations are summarized in Table 1.

The spectral observations were reduced with the 4A software system (Ilyin 2000). Bias, cosmic ray, flat field and scattered light corrections, wavelength calibration and normalization were included in the reduction process.

The stellar parameters used in the inversions were chosen according to Berdyugina et al. $(1998 \mathrm{a})$, and read $T_{\text {eff }}=4600 \mathrm{~K}, \xi_{t}=2.4 \mathrm{kms}^{-1}, \zeta_{t}=3.5 \mathrm{kms}^{-1}, P_{\text {orb }}=6.724333 \mathrm{~d}$, $v \sin i=22.6 \mathrm{kms}^{-1}, i=60^{\circ}$.

\section{Results and conclusions}

The following spectral lines were used in the surface temperature inversions: Fe I 6173 A, Ni I $6175 \AA$, Ni I $6177 \AA$, and Fe I $6180 \AA$. Spectral line parameters were obtained 

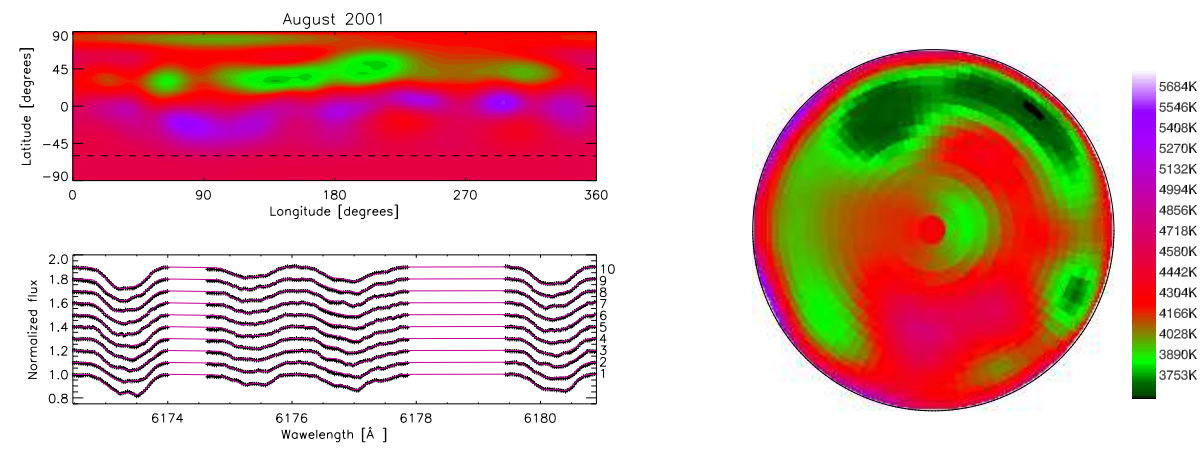

Figure 3. Observing run during August 2001.
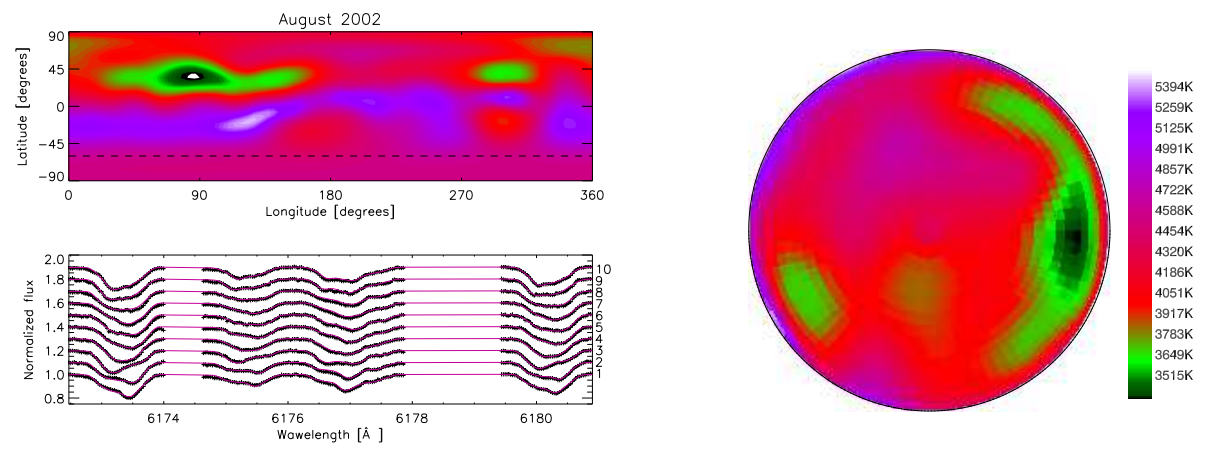

Figure 4. Observing run during August 2002.


Figure 5. Observing run during November 2002.

from the Vienna Atomic Line Database (Kupka et al. 1999); the $\log (g f)$ values of the two included Ni I lines were modified from the standard value of -0.53 to -0.2 as the standard values were found to produce much weaker absorption lines than the actually observed ones. This is equivalent to an increase in the $\mathrm{Ni}$ abundancy, which is probably the real reason for the observed strong Ni lines.

Figures 1-5 show the results of the inversions. During July-August 1999 (Fig. 1) only one spot is seen approximately at latitude $40^{\circ}$. The minimum temperature inside the spot was measured to occur at the approximate longitude of $170^{\circ}$ or phase 0.47 . Our image is quite different from the one obtained by $\mathrm{Gu}$ et al. (2003) for almost a simultane- 


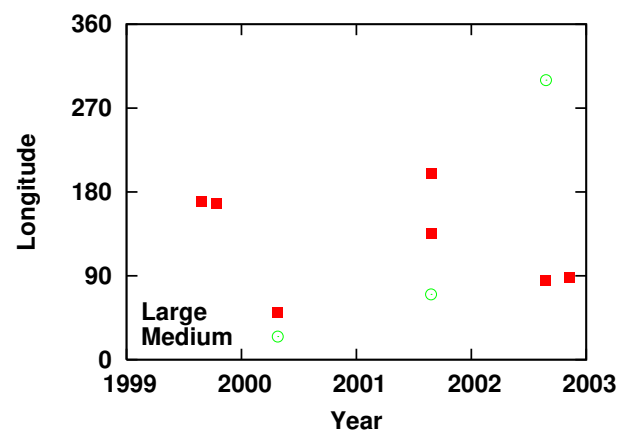

Figure 6. The spot longitudes of II Peg versus time. Square symbols represent larger active regions and circles mark weak structures.

ous observing period, but a different spectral region. Their inversions gave much larger, longitudinally extended, spot structures around $170-290^{\circ}$ at roughly $60^{\circ}$ of latitude.

In September 1999 our inversions also show only one strong spot that has barely moved in the orbital reference frame (latitude $44^{\circ}$ and longitude $160^{\circ}$ or phase 0.44 ). There is a weaker, longitudinally extended feature visible between $220-270^{\circ}$ or phase $0.61-0.75$.

Almost two years later, in August 2001 (Fig. 3), the star exhibits much more surface structures: three spots are visible in our image (longitudes $70^{\circ}, 140^{\circ}$, and $200^{\circ}$ or phases $0.19,0.39$ and 0.56 ) at an approximate latitude of $40^{\circ}$. The inversions of $\mathrm{Gu}$ et al. (2003) for an observing run 5 months later show much less structure, and the maximal activity seems to have moved roughly to the other side of the star than what was seen in their images during 1999 and 2000.

One year later, in August 2002 (Fig. 4) our maps show one strong spot at the latitude $40^{\circ}$ and longitude $80^{\circ}$ or phase 0.22 , and a weaker one at $300^{\circ}$ or phase 0.83 . In November 2002 (Fig. 5) only the stronger spot is seen approximately at the same location. Comparing the surface temperature distribution during the observing seasons 1999 and 2002, the maximal spot activity seems to have moved by roughly $100^{\circ}$ in the orbital reference frame, while very little drift of the spots can be seen during the consequtive observing runs during 1999 and 2002. In between these two 'states' of only one strong spot at different location on the surface, a much more complex distribution could be seen during August 2001. Our images give some support to the conclusion of $\mathrm{Gu}$ et al. (2003) of a significant change of the longitudinal spot distribution having occured sometime during 2001 (Fig. 6).

We plan to continue to study the spot evolution on II Peg by analysing photometric and spectropolarimetric observations of the object, both to invert the surface temperature distribution, but also the magnetic field configuration of the object.

\section{Acknowledgements}

The authors acknowledge the hospitality of NORDITA during the programme 'Solar and stellar dynamos and cycles', and useful discussions with Dr. Oleg Kochukhov

\section{References}

\section{References}

Berdyugina, S.V., Berdyugin, A.V., Ilyin, I., Tuominen, I. 1998a, A\& A, 340, 437 
Berdyugina, S.V., Jankov, S., Ilyin, I., Tuominen, I., Fekel, F.C. 1998b, A\& A, 334

Berdyugina, S.V., Tuominen, I. 1998c, A\&A, 336, L25

Berdyugina, S.V., Ilyin, I., Tuominen, I. 1999a, A\&A, 347, 932

Berdyugina, S.V., Ilyin, I., Tuominen, I. 1999b, A\&A, 349, 863

Berdyugina, S.V., Berdyugin, A.V., Ilyin, I., Tuominen, I. 1999c, ApJ, 350, 626

Carroll, T., Kopf, M., Strassmeier, K., Ilyin, I., Tuominen, I. 2009, IAU Symposium, 259

Gu, S.-H., Tan, H.-S., Wang, X.-B., Shan, H.-G. 2003, A\&A, 405, 763

Hackman, T., Jetsu, L., Tuominen, I. 2001, A\&A, 374, 171

Ilyin, I. 2000, PhD thesis, University of Oulu, Finland

Kupka, F., Ryabchikova, T.A. 1999, VALD-The Vienna Atomic Line Database: A Survey

Kurster, M. 1993, A\&A A, 274, 851

Kurucz, R.L. 1993, VizieR On-line Data catalog: VI/39

Piskunov, N.E., Tuominen, I., Vilhu, O. 1990, A\&AA, 230, 363

Piskunov, N.E. 1991, Proceedings of Colloquium No. 130 of the International Astronomical Union

Rodonò, M., Messina, S., Lanza, A.F., Cutispoto, G., Teriaca, L. 2000, A\&A, 358, 624 